
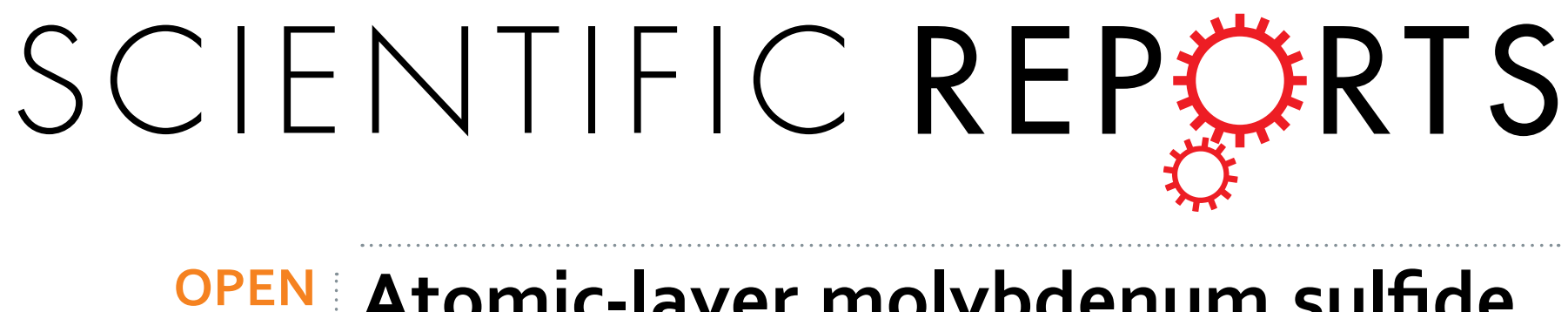

\title{
Atomic-layer molybdenum sulfide optical modulator for visible coherent light
}

Received: 19 March 2015

Accepted: 19 May 2015

Published: 12 June 2015

\author{
Yuxia Zhang ${ }^{1}$, Shuxian Wang ${ }^{1}$, Haohai Yu ${ }^{1}$, Huaijin Zhang ${ }^{1}$, Yanxue Chen ${ }^{2}$, Liangmo Mei ${ }^{2}$, \\ Alberto Di Lieto ${ }^{3}$, Mauro Tonelli3 \& Jiyang Wang ${ }^{1}$
}

Coherent light sources in the visible range are playing important roles in our daily life and modern technology, since about $50 \%$ of the capability of the our human brains is devoted to processing visual information. Visible lasers can be achieved by nonlinear optical process of infrared lasers and direct lasing of gain materials, and the latter has advantages in the aspects of compactness, efficiency, simplicity, etc. However, due to lack of visible optical modulators, the directly generated visible lasers with only a gain material are constrained in continuous-wave operation. Here, we demonstrated the fabrication of a visible optical modulator and pulsed visible lasers based on atomiclayer molybdenum sulfide $\left(\mathrm{MoS}_{2}\right)$, a ultrathin two-dimensional material with about 9-10 layers. By employing the nonlinear absorption of the modulator, the pulsed orange, red and deep red lasers were directly generated. Besides, the present atomic-layer $\mathrm{MoS}_{2}$ optical modulator has broadband modulating properties and advantages in the simple preparation process. The present results experimentally verify the theoretical prediction for the low-dimensional optoelectronic modulating devices in the visible wavelength region and may open an attractive avenue for removing a stumbling block for the further development of pulsed visible lasers.

Visual information from our eyes goes to our brains and then is processed ${ }^{1-3}$. It has been well-understood that nearly $50 \%$ of the capability of our brains is devoted to processing visual information ${ }^{2,3}$. Therefore, besides the numerous applications in our daily life, including entertainment, education and medical treatment, visible lasers also have important requirements in modern science and technologies ${ }^{1,7}$. Nonlinear optics and direct lasing of gain materials ${ }^{4-6}$ are the techniques for getting the visible lasers, beside the visible laser diodes which have relatively poor coherence ${ }^{8,9}$. In contrast to lasers achieved by nonlinear frequency-shifting of infrared lasers ${ }^{10}$, direct lasing has the advantages in the aspects of compactness, efficiency, simplicity, etc. In the laser regime, pulsed lasers with fast- or ultrafast pulses and high- or ultra-high peak power provide a platform for the studying ultrafast kinetics process ${ }^{11,12}$, realizing strong field physics ${ }^{13,14}$, etc., and is an vital scientific topic in lasers. A optical modulator for tuning the gain or loss in the oscillator is a crucial device for the generation of pulses. However, constrained by optical modulators, especially passive optical modulators, the directly generated visible pulses are rare up to now ${ }^{15,16}$. In theory, the optical modulators in visible lasers can be realized by semiconductor saturable absorber mirrors ${ }^{17}$, but they require complexly and elaborately designed quantum wells, besides their narrow response wavelength band.

Recent years, atomic-layered transition-metal dichalcogenides have emerged as promising next-generation optoelectronic materials, since their exceptional and interesting electronic and photonic properties $^{18-23}$. Their strong nonlinear optical response, including the second and third nonlinearities are also remarkable ${ }^{21,24}$. Based on the nonlinear absorption and band-gap engineering, the transition-metal

${ }^{1}$ State Key Laboratory of Crystal Materials and Institute of Crystal Materials, Shandong University, Jinan 250100, China. ${ }^{2}$ School of Physics, Shandong University, Jinan 250100, China. ${ }^{3 N E S T}$ Istituto Nanoscienze-CNR and Dipartimento di Fisica dell'Università di Pisa, Largo B. Pontecorvo 3, 56127 Pisa, Italy. Correspondence and requests for materials should be addressed to H.Y. (email: haohaiyu@sdu.edu.cn) or H.Z. (email: huaijinzhang@sdu.edu.cn) 

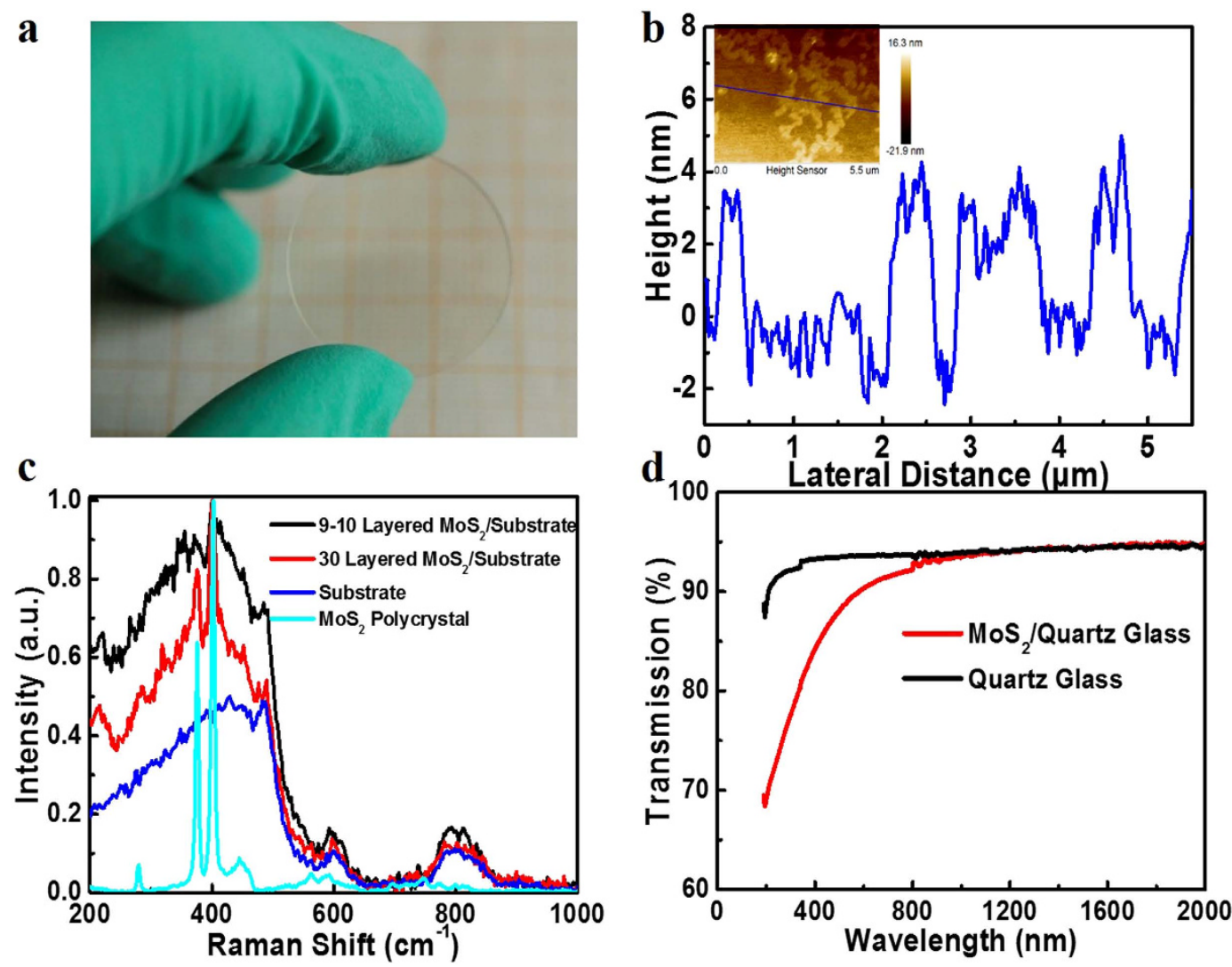

Figure 1. Characterization of prepared MoS2 thin film. (a) Prepared 9-10 layered $\mathrm{MoS}_{2}$ sample on a quartz glass substrate with the dimensions of $\Phi 25 \mathrm{~mm} \times 1 \mathrm{~mm}$ ( $\Phi$ is the wafer diameter). (b) Typical variation of $\mathrm{MoS}_{2}$ thin film height. Inset: Morphology of sand-abraded $\mathrm{MoS}_{2}$ thin film measured with atomic force microcopy. (c) Raman spectra of prepared 9-10 layered $\mathrm{MoS}_{2}$ /quartz glass sample, 30 layered $\mathrm{MoS}_{2} /$ quartz glass sample, quartz substrate, and polycrystalline $\mathrm{MoS}_{2}$. (d) Absorption spectra of prepared $\mathrm{MoS}_{2} /$ quartz glass sample and quartz glass substrate.

dichalcogenides saturable absorbers were developed as broadband optical modulators for pulsed laser operation in the wavelength range from $1 \mu \mathrm{m}$ to $2 \mu \mathrm{m}^{21,25}$. However, maybe associated to the large photon energy carried by the visible laser and the band-gap of present low-dimensional optoelectronic materials, the applications of low-dimensional optoelectronic materials, including, carbon nanotubes, graphene, topological insulators and transition metal dichalcogenides, in visible lasers as a modulating device mainly exist in theoretical prediction ${ }^{21,26-29}$. Considering the nonlinear optical properties of the low-dimensional optoelectronic materials, few-layered $\mathrm{MoS}_{2}$ has large nonlinearity and saturable intensity, 5 orders of magnitude larger than graphene in the visible wavelength range ${ }^{21,25,27}$, which theoretically indicates that few-layered $\mathrm{MoS}_{2}$ should be a promising optical modulator for the generation of pulsed visible laser with large pulse energy. Here, we report optical modulation of visible coherent light by less than 10 layers $\mathrm{MoS}_{2}$ in the wavelength band from orange to deep red. Based on the visible $\mathrm{MoS}_{2}$ optical modulator, the directly generated pulsed visible lasers were demonstrated.

\section{Results}

Raman, atomic force microscopy and spectral analysis of $\mathrm{MoS}_{\mathbf{2}}$ film. The $\mathrm{MoS}_{2}$ film was prepared with pulsed laser deposition (PLD) with a polished optical-grade far-ultraviolet quartz glass wafer $\left(\Phi 25 \mathrm{~mm} \times 1 \mathrm{~mm}, \Phi\right.$ is the wafer diameter) which can provide high resolution in the visible range ${ }^{21}$. The prepared sample is shown in Fig. 1a. In order to accurately measure the number of layers, the prepared $\mathrm{MoS}_{2}$ samples were investigated with atomic force microscopy (AFM) by abrading with sand. The change of the $\mathrm{MoS}_{2}$ height is provided in Fig. 1b which shows that the height change is about $5.8 \mathrm{~nm}$ and the $\mathrm{MoS}_{2}$ film is about 9-10 layers on the substrate since the $\mathrm{MoS}_{2}$ layers were assumed to bond together via the Van der Waals' interaction ${ }^{30}$. The 9-10 layered $\mathrm{MoS}_{2}$ sample was characterized with a Raman spectrometer and the Raman shifting is displayed in Fig. 1c. For comparison, the 30 layered $\mathrm{MoS}_{2}$ thin film and polycrystalline $\mathrm{MoS}_{2}$ are also presented in this figure. From this figure, it can found that for the 9-10 layered $\mathrm{MoS}_{2}$ thin film, an in-plane $\mathrm{E}_{2 \mathrm{~g}}{ }^{1}$ vibrational mode and an out-of-plane $\mathrm{A}_{1 \mathrm{~g}}$ vibrational mode appear at $378 \mathrm{~cm}^{-1}$ and $400 \mathrm{~cm}^{-1}$, respectively. The $A_{1 g}$ mode also shows a shift to larger wavenumbers 
with the increase of layers, which is in agreement with the previous reported anomalous lattice vibrations of layered $\mathrm{MoS}_{2}{ }^{31}$.

For determining the responding wavelength range of the prepared $\mathrm{MoS}_{2}$ sample, the transmission spectrum was measured with a V-570 JASCO UV/VIS/NIR spectrophotometer from the wavelength of $190 \mathrm{~nm}$ and is shown in Fig. 1d. For comparison, the spectrum of a quartz glass substrate is also given. From this figure, it can be observed that the transmission increases with the increase of the wavelength, changes smooth when the wavelength is larger than $1000 \mathrm{~nm}$ and displays the prepared sample becoming indistinguishable with the substrate when the wavelength is larger than $1250 \mathrm{~nm}$. The results indicate that the band gap of the prepared sample is about $1 \mathrm{eV}$ in the range of $0.86-1.29 \mathrm{eV}$ of multilayered $\mathrm{MoS}_{2}{ }^{32,33}$ and also in agreement with the theoretical calculation of about 9-10 layered $\mathrm{MoS}_{2}{ }^{34}$. It should be noted that in the visible range from $380 \mathrm{~nm}$ to $780 \mathrm{~nm}$, the transmission of the prepared $\mathrm{MoS}_{2} /$ quartz glass sample increases from $83.2 \%$ to $92.1 \%$ with augmenting the wavelength. Therefore, we can get the conclusion that all the visible photons can transfer the electrons from the valence band to the conduction band of the prepared sample and the prepared $\mathrm{MoS}_{2}$ sample has response in all the visible range.

Based on the measurement shown in Fig. 1 and the previous reports on the saturable absorption properties of few- and multi-layered $\mathrm{MoS}_{2}{ }^{21,25}$, it can be gotten the conclusion that under strong excitation with the wavelength shorter than $1250 \mathrm{~nm}$, the electronic states could be fully occupied due to the transfer of electrons from the valence band to conduction band, so that the absorption of the prepared $\mathrm{MoS}_{2}$ thin film would be saturated. The kinetic process of absorption can be described by the power-dependent transmission formula of two-level absorber $\mathrm{as}^{35}$ :

$$
T=A \exp \left(\frac{-\delta T}{1+\frac{I}{I_{\text {sat }}}}\right)
$$

where $T$ is the transmission of the $M_{2}$ thin film, $A$ is a normalization constant, $\delta T$ is the absolute modulation depth of the sample, $I$ is the incident intensity and $I_{\text {sat }}$ is the saturation intensity. Considering the previous investigations on few-layered $\mathrm{MoS}_{2}$ thin film ${ }^{21,25}$, we can get the results that the saturation intensity $I_{\text {sat }}$ should be in the range from $136 \mathrm{GW} / \mathrm{cm}^{2}$ to $280 \mathrm{GW} / \mathrm{cm}^{2}$ in the visible wavelength from $400 \mathrm{~nm}$ to $800 \mathrm{~nm}$.

Optical modulating properties of $\mathrm{MoS}_{2}$ film in the visible range and pulsed visible lasers. Praseodymium $\left(\mathrm{Pr}^{3+}\right)$ ions in a suitable crystalline field can emit the visible light from green to deep red wavelengths and $\operatorname{Pr}^{3+}$ lasers are the representative in the visible laser regime ${ }^{6}$. With a 1.01 at.\% praseodymium doped lithium fluoride gadolinium crystal $\left(\mathrm{Pr}: \mathrm{GdLiF}_{4}\right)$ as the gain materials and a laser diode with a emission wavelength centered at $445 \mathrm{~nm}$ as the pump source, the optical modulating properties and application of the $\mathrm{MoS}_{2}$ sample in the laser at orange $(605 \mathrm{~nm})$, red $(639 \mathrm{~nm})$ and deep red $(721 \mathrm{~nm})$ wavelengths were investigated and the pulsed visible lasers were realized. In the laser experiments, different cavities were built for realizing different wavelength lasers as described in Methods section and the prepared $\mathrm{MoS}_{2}$ thin film was used as the universal pulse modulator for the generation of all the visible laser pulses. The laser operation was controlled by inserting or removing the $\mathrm{MoS}_{2}$ thin film in the cavity. The continuous and Q-switched laser output power were measured by a power meter. The pulsed output performance including the repetition rate and pulse width was monitored by a digital oscilloscope and a silicon detector.

By removing $\mathrm{MoS}_{2}$ from the cavity, the continuous-wave (CW) visible lasers were realized and the results were shown in Fig. 2a-c at the wavelengths of $605 \mathrm{~nm}, 639 \mathrm{~nm}$ and $721 \mathrm{~nm}$, respectively. By inserting the $\mathrm{MoS}_{2}$ modulator, the corresponding pulsed lasers were achieved and were also presented in Fig. 2a-c for comparison with the CW lasers. From the figures shown above, we found that all the output power, including CW and pulsed lasers, increases with the increase of pump power. However, with the increase of pump power over than a threshold, the pulse performance became unstable due to the generation of CW components in the pulsed lasers which means that under this threshold, the loss modulation of the $\mathrm{MoS}_{2}$ thin film can switch the lasers completely based on the analysis on the passive modulating ${ }^{35}$. This threshold is related to the gain of the oscillating intensity in the cavity and the absorption of the modulator. Since the gain of the laser crystal and the absorption of the $\mathrm{MoS}_{2}$ thin film at presented wavelengths are different and the transmission of output mirrors is low ( $4 \%$ at $605 \mathrm{~nm}, 1.8 \%$ at $639 \mathrm{~nm}$ and $1.4 \%$ at $721 \mathrm{~nm}$ ), the thresholds are changeable with the value of $1.58 \mathrm{~W}, 0.98 \mathrm{~W}$ and $1.26 \mathrm{~W}$ at the $605 \mathrm{~nm}, 639 \mathrm{~nm}$ and $721 \mathrm{~nm}$ lasers, respectively. The maximum CW output power is $248 \mathrm{~mW}, 430 \mathrm{~mW}$ and $232 \mathrm{~mW}$, and the maximum pulsed average output power is $17 \mathrm{~mW}, 9 \mathrm{~mW}$ and $8 \mathrm{~mW}$ at the wavelength of $605 \mathrm{~nm}, 639 \mathrm{~nm}$ and $721 \mathrm{~nm}$, respectively.

The repetition rate and pulse width with the increase of the pump power at different wavelength are shown in Fig. $2 \mathrm{~d}-\mathrm{f}$, respectively. It should be noted that the repetition rate displays an increasing tendency with the pump power. However, the repetition rate shows a knee point under the pump power of $1.53 \mathrm{~W}$ at the wavelength of $605 \mathrm{~nm}$, which should be related to the high-order absorption including the carrier or two-photon absorption ${ }^{24}$ and the high photon energy carried by this wavelength laser. The maximum repetition rate is $246 \mathrm{kHz}, 210 \mathrm{kHz}$ and $177 \mathrm{kHz}$ at the wavelength of $605 \mathrm{~nm}, 639 \mathrm{~nm}$ and $721 \mathrm{~nm}$, respectively. The pulse widths decrease with the increase of incident pump power, which is 
a
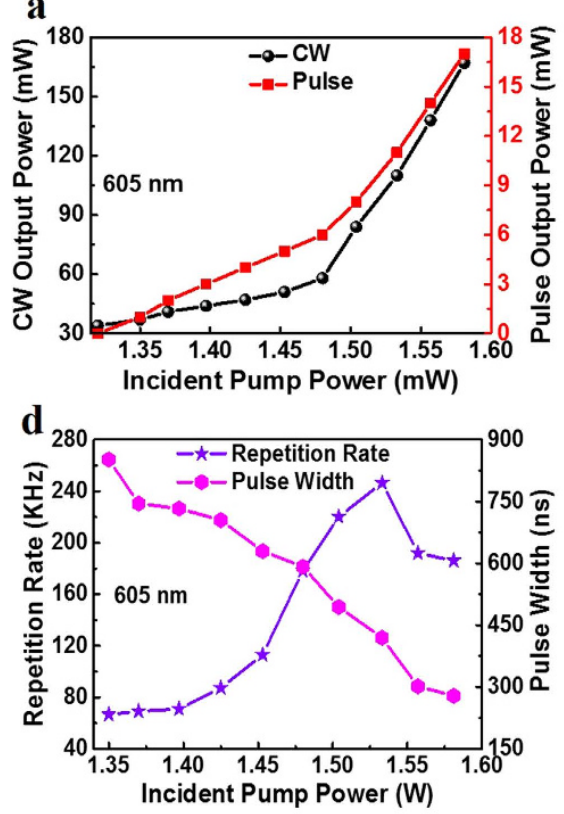

b
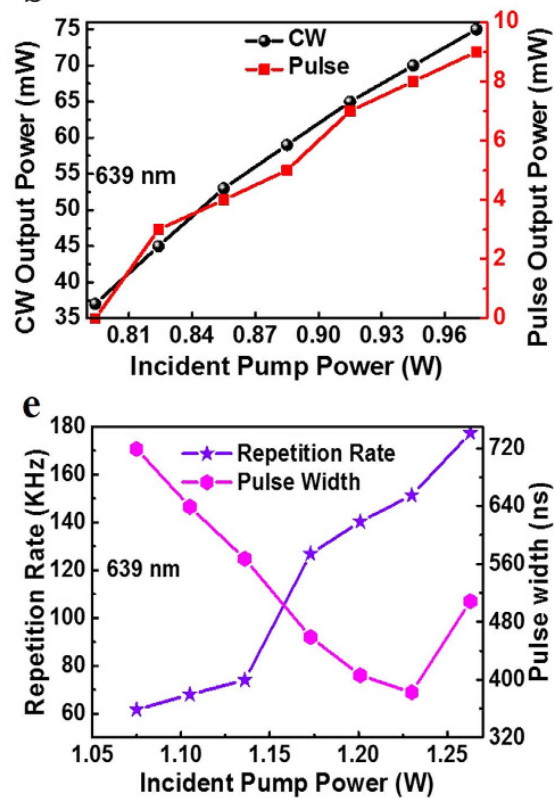
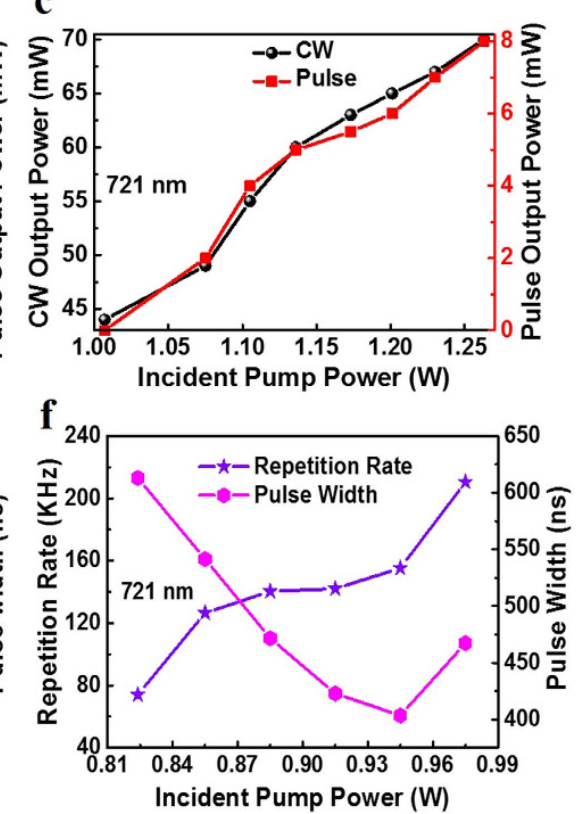

Figure 2. Pulsed visible laser performance with the prepared MoS2 as a modulator. (a)-(c) Continuouswave and pulsed laser output power at the wavelength of $605 \mathrm{~nm}$ (orange), $639 \mathrm{~nm}$ (red) and $721 \mathrm{~nm}$ (deep red), respectively. (d)-(f) Repetition rate and pulse width of the pulsed laser at the wavelength of $605 \mathrm{~nm}$ (orange), $639 \mathrm{~nm}$ (red) and $721 \mathrm{~nm}$ (deep red), respectively.

typical for the passively modulated lasers ${ }^{21,36}$. The minimum pulse width at the wavelength of $605 \mathrm{~nm}$, $639 \mathrm{~nm}$ and $721 \mathrm{~nm}$ is $278 \mathrm{~ns}, 403 \mathrm{~ns}$ and $382 \mathrm{~ns}$, respectively. With the average output power and repetition rate, the maximum pulse energy at the wavelength of $605 \mathrm{~nm}, 639 \mathrm{~nm}$ and 721 is $91 \mathrm{~nJ}, 52 \mathrm{~nJ}$ and $68 \mathrm{~nJ}$, respectively. Combing the pulse energy and pulse width, the maximum peak power at the wavelength of $605 \mathrm{~nm}, 639 \mathrm{~nm}$ and $721 \mathrm{~nm}$ is $327 \mathrm{~mW}, 127 \mathrm{~mW}$ and $120 \mathrm{~mW}$, respectively. Representative pulse trains at the wavelengths of $605 \mathrm{~nm}, 639 \mathrm{~nm}$ and $721 \mathrm{~nm}$ are shown in Fig. $3 \mathrm{a}-\mathrm{c}$, with the repetition of $69.1 \mathrm{kHz}, 140.3 \mathrm{kHz}$, and $74 \mathrm{kHz}$, respectively. As shown in the figure, the pulses are uniformly spaced and the pulsed lasers are stable. With an optical spectrum analyzer (HR4000, Ocean Optics Inc), the pulsed laser spectra are recorded and shown in Fig. $3 \mathrm{~d}-\mathrm{f}$, whose peaks are located at $605 \mathrm{~nm}, 639 \mathrm{~nm}$ and $721 \mathrm{~nm}$, respectively.

\section{Discussions}

From the discovery of lasers, visible lasers play important roles in many fields. However, the pulsed visible lasers are mainly achieved by the nonlinear optical process and the development of the directly generated pulsed visible lasers are constrained by rare suitable modulators. Since the first reported carbon nanotube pulsed modulator ${ }^{26}$, it has been theoretically predicted that low-dimension optoelectronic materials including carbon nanotube, graphene, topological insulators, and $\mathrm{MoS}_{2}$ could be used as pulses modulators in the visible lasers. However, up to now, no any experimental results were reported in this wavelength range. Beside strong nonlinear optical properties, $\mathrm{MoS}_{2}$ has a band gap in the range of 0.86 $\mathrm{eV}$ to $1.8 \mathrm{eV}$ changeable with the layer number ${ }^{34}$, which means that this material should be a promising visible laser modulator. Aiming at the possible application in visible pulsed lasers, the 9-10 layered $\mathrm{MoS}_{2}$ thin film was prepared with the PLD method on a quartz-glass substrate. By investigating its transmission spectra and based on the previous investigation on its electronic structure and nonlinear optics $^{21,24,34}$, we found that this prepared sample could response to the photons at the wavelength less than $1250 \mathrm{~nm}$, especially in the visible range as a pulse modulator.

In order to verify the pulse modulating properties of $\mathrm{MoS}_{2}$ samples in the visible wavelength, the prepared sample was inserted into a simple directly generated visible laser cavity to be used as a pulse modulator. The pulsed lasers in the wavelength ranging from $605 \mathrm{~nm}$ to $721 \mathrm{~nm}$ were realized and stable pulses were achieved and analyzed, which shows that the two-dimensional $\mathrm{MoS}_{2}$ has excellent modulating properties in the visible range. Besides, considering the low dimension, it could be proposed that this device would also have some promising applications in the micro- and nano-optoelectronics. For future improvement of the $\mathrm{MoS}_{2}$ pulsed laser performance, the laser cavity and the $\mathrm{MoS}_{2}$ layers number should be optimized for satisfying different requirement. Furthermore, these results also experimentally verify the theoretical prediction about the applications of the two-dimensional optoelectronic materials in visible lasers as a modulator. 

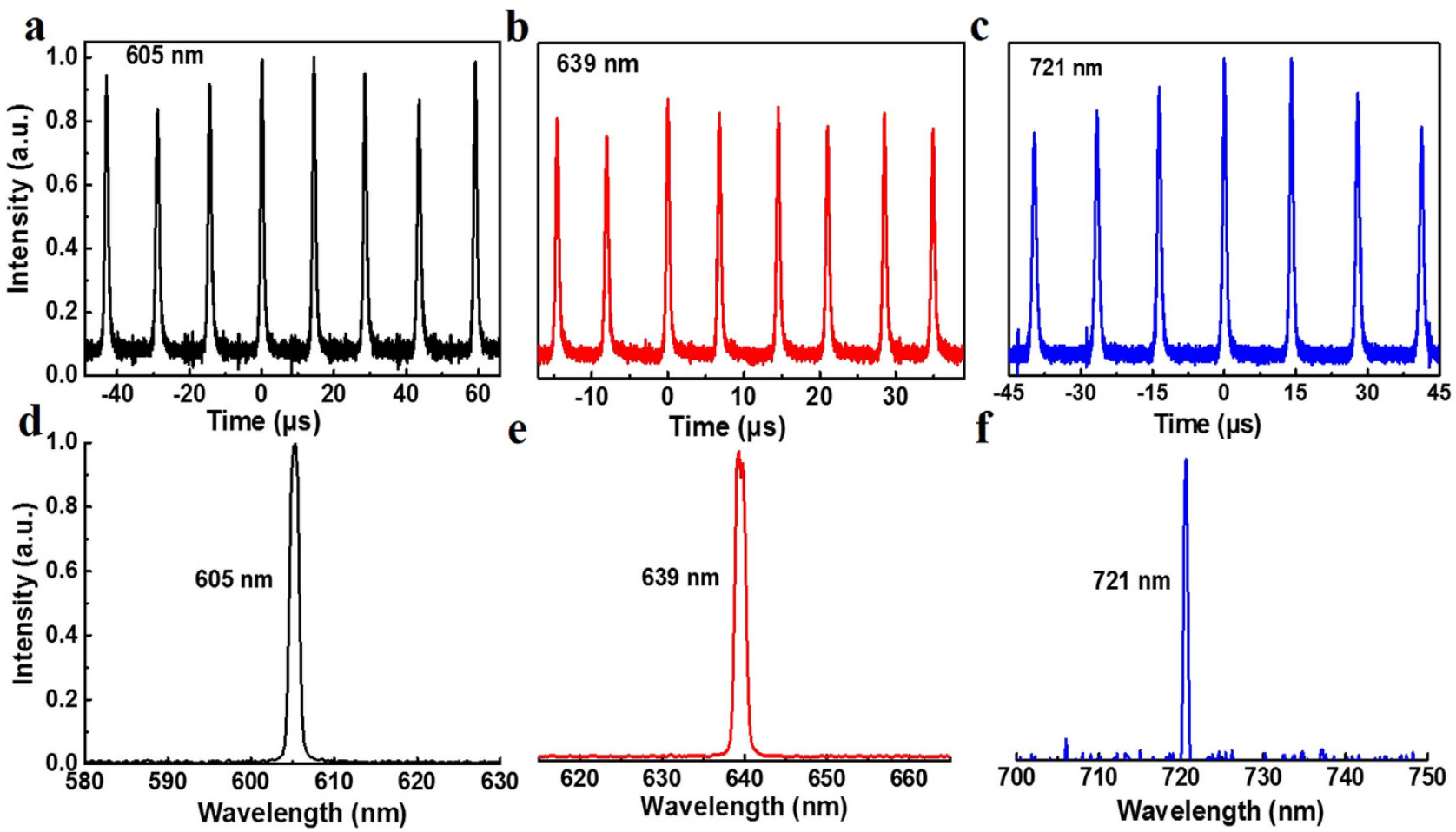

Figure 3. Typical pulse trains and spectra of pulsed visible lasers. (a)-(c) Pulse train with the repetition rate of $69.1 \mathrm{kHz}$ at the wavelength of $605 \mathrm{~nm}, 140.3 \mathrm{kHz}$ at the wavelength of $639 \mathrm{~nm}$, and $74 \mathrm{kHz}$ at the wavelength of $721 \mathrm{~nm}$, respectively. (d) $-(\mathbf{f})$ Pulsed laser spectrum with the center wavelength of $605 \mathrm{~nm}$, $639 \mathrm{~nm}$ and $721 \mathrm{~nm}$, respectively.

In conclusion, we provide an atomic-layer $\mathrm{MoS}_{2}$ optical modulating device for the visible lasers. The responding wavelength regime of the prepared device was investigated. Based on the device, pulsed orange, red and deep red lasers were realized which presents that the prepared device is a promising optical modulator. The results also widen the application wavelength range of two-dimensional optoelectronic materials as modulators from the well-reported infrared wavelength to the rare visible wavelength. Additionally, the present results should also be helpful for the development of lasers especially for the pulsed visible lasers which are constrained by the suitable modulator up to now. To sum up, our findings may constitute the basis for the further development of pulsed visible lasers and open a new door for the two-dimensional optoelectronic materials.

\section{Methods}

Preparation of atomic-layer $\mathrm{MoS}_{2}$ optical modulator. The atomic-layer $\mathrm{MoS}_{2}$ thin film was prepared by the pulsed laser deposition (PLD) technique ${ }^{21,37}$ with polycrystalline $\mathrm{MoS}_{2}$ powder as the raw materials. A commercial optical-grade far-ultraviolet quartz glass wafer $\left(\Phi 25 \times 1 \mathrm{~mm}^{3}\right)$ was chosen as the substrate which has high-transmission in the visible wavelength range. By cold pressing at about $70 \mathrm{MPa}, \mathrm{MoS}_{2}$ powder was pressed into a $40 \mathrm{~mm}$ diameter pellet. The excitation source is a Comppex Pro $201 \mathrm{KrF}$ excimer laser (provided by Coherent Inc.) at a wavelength of $248 \mathrm{~nm}$ and a pulse width of $20 \mathrm{~ns}$, which provide the radiation and ablation to the target in a spot area of about $5 \mathrm{~mm}^{2}$. The repetition rate of the $\mathrm{KrF}$ excimer laser is $5 \mathrm{~Hz}$ with the pulse energy of $600 \mathrm{~mJ} /$ pulse (corresponding to an energy density of $8.5 \mathrm{~J} / \mathrm{cm}^{2}$ ). The base pressure of the vacuum chamber system was about $8.9 \times 10^{-5} \mathrm{~Pa}$, and in the deposition process, the pressure is up to about $5 \times 10^{-4} \mathrm{~Pa}$. Both of the $\mathrm{MoS}_{2}$ target and quartz-glass substrate can be rotated for ensuring the uniformity of the prepared film and the temperature of the substrate was fixed at $300^{\circ} \mathrm{C}$. The number of layers can be controlled by the deposition time.

Laser configuration with $\mathrm{MoS}_{2}$ as the pulse modulator. The pulsed visible laser cavity is shown in Fig. 4. In all the visible laser experiments, a laser diode with a central wavelength of $445 \mathrm{~nm}$ was used as the pump source and a Pr:GdLiF 4 crystal was employed as the gain material. The laser cavity is plane-concave formed by $\mathrm{M} 1$ and $\mathrm{M} 2$. With a $\mathrm{f}=25 \mathrm{~mm}$ lens, the pump light was focused into the Pr:GdLiF ${ }_{4}$ crystal by passing through the input plane mirror M1, antireflection (AR) coated for the pump wavelength of $445 \mathrm{~nm}$ and highly reflective (HR) for the laser wavelength $(550-780 \mathrm{~nm})$. The gain material cut along the $a$ direction has the dimensions of $2.5 \mathrm{~mm} \times 2 \mathrm{~mm} \times 6.9 \mathrm{~mm}(a \times c \times a)$ and both of the 


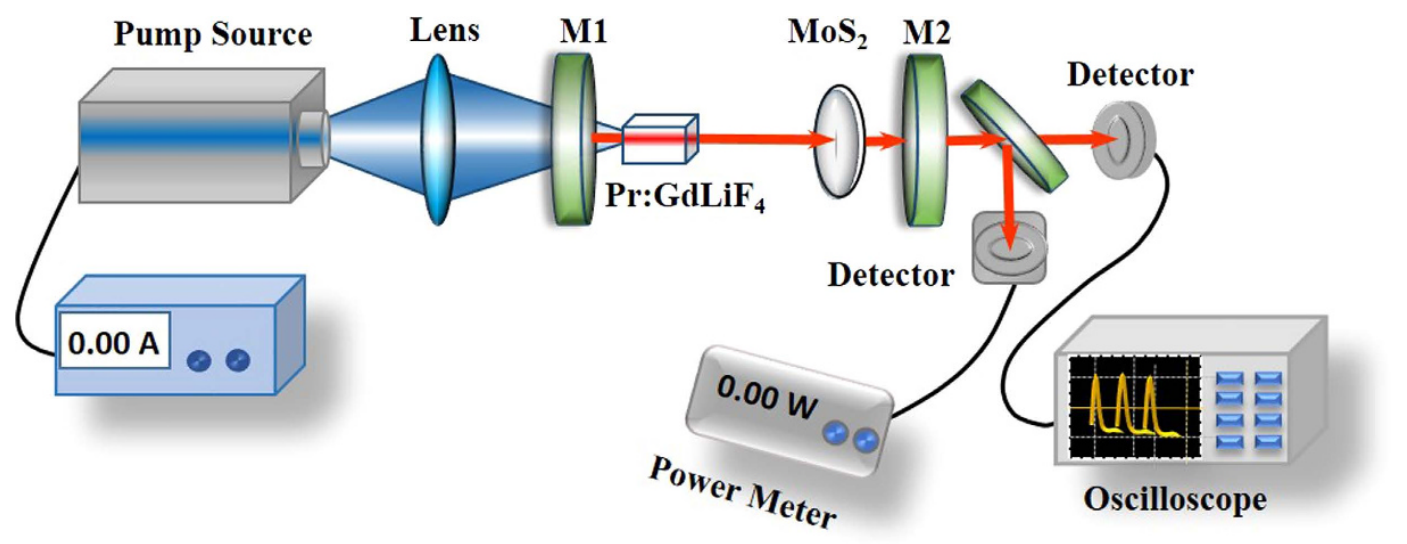

Figure 4. Configuration of the pulsed visible lasers modulated by the prepared MoS2 thin film.

end faces were polished. In order to removing the heat generated during the laser process, the gain was wrapped by an indium foil and mounted in a copper block cooled by $7{ }^{\circ} \mathrm{C}$ water. The optimized length of the cavity was optimized to be about $45 \mathrm{~mm}$. By changing the output couplers $\mathrm{M} 2$, the orange, red and deep red lasers were obtained at the wavelengths of $605 \mathrm{~nm}, 639 \mathrm{~nm}$, and $721 \mathrm{~nm}$, respectively. All the output couplers $\mathrm{M} 2$ have the radii of curvature of $50 \mathrm{~mm}$. For the orange laser, the output coupler M2 has the transmissivity of $4 \%$ at $605 \mathrm{~nm}$ and high transmission at 620 to $721 \mathrm{~nm}$ for suppressing the oscillating at red and deep red light. For the red laser, the output coupler M2 has the transmissivity of $1.8 \%$ at $639 \mathrm{~nm}$ and high transmission at $721 \mathrm{~nm}$. For the deep red laser, the output coupler M2 has the transmissivity of $1.4 \%$ at $721 \mathrm{~nm}$ and high transmission at the wavelength under $700 \mathrm{~nm}$. In the pulsed lasers, the $\mathrm{MoS}_{2}$ /quartz-glass modulator was insert into the cavity as close as possible to the output coupler. The continuous and Q-switched laser output power were measured by a power meter. The pulsed output performance including the repetition rate and pulse width was monitored by a digital oscilloscope and a silicon detector.

\section{References}

1. Conchello, J. A. \& Lichtman, J. W. Optical sectioning microscopy. Nat. Methods 2, 920-931 (2005).

2. Marieb, E. N. \& Hoehn, K. N. Human Anatomy \& Physiology (Pearson, 2012).

3. Geng, J. Three-dimensional display technologies. Adv. Opt. Photon. 5, 456-535 (2013)

4. Franken, P. A., Hill, A. E., Peters, C. W. \& Weinreich, G. Generation of Optical Harmonics. Phys. Rev. Lett. 7, 118-119 (1961)

5. Esterowitz, L. et al. Blue light emission by a Pr:LiYF -laser operated at room temperature. J. Appl. Phys. 48, 650-652 (1977).

6. Huber, G., Kränkel, C. \& Petermann, K. Solid-state lasers: status and future. J. Opt. Soc. Am. B 27, B93-B105 (2010).

7. Baumgarth, N. \& Roederer, M. A practical approach to multicolor flow cytometry for immunophenotyping. J. Immunol. Methods 243, 77-97 (2000).

8. Hall, R. N., Fenner, G. E., Kingsley, J. D., Soltys, T. J. \& Carlson, R. O. Cohenent light emission from GaAs junctions. Phys. Rev. Lett. 9, 366-368 (11962).

9. Zeng, X. et al. Solitary pulse-on-demand production by optical injection locking of passively Q-switched InGaN diode laser near lasing threshold. Appl. Phys. Lett. 106, 071101-5 (2015).

10. Armstrong, J. A., Blgemeergen, N., Ducuing, J. \&Pershan, P. S. Interactions between light waves in a nonlinear dielectric. Phys. Rev. 127, 1918-1939 (1972).

11. Zewail, A. H. Femtochemistry. Past, present, and future. Pure and Appl. Chem. 72, 2219-2231 (2000).

12. Carlsson, J., Jönsson, P. \& Sturesson, L. Accurate time-resolved laser spectroscopy on silver atoms. Z. Phys. D: At. Mol. Clust. 16, 87-90 (1990).

13. Hurricane, O. A.et al. Fuel gain exceeding unity in an inertially confined fusion implosion. Nat. 506, 343-348 (2014).

14. Kawata, S., Sun, H. B., Tanaka, T. \& Takada, K. Finer features for functional microdevices. Nat. 412, 697-698 (2001).

15. Gaponenko, M. et al. SESAM mode-locked red praseodymium laser. Opt. Lett. 39, 6939-6941 (2014).

16. Abe, R., Kojou, J., Masuda, K. \& Kannari, F. Cr ${ }^{4+}$-doped $\mathrm{Y}_{3} \mathrm{Al}_{5} \mathrm{O}_{12}$ as a saturable absorber for a Q-switched and mode-locked 639-nm $\mathrm{Pr}^{3+}$-doped $\mathrm{LiYF}_{4}$ laser. Appl. Phys. Express 6, 032703 (2013).

17. Keller, U. Semiconductor saturable absorber mirrors (SESAM's) for femtosecond to nanosecond pulse generation in solid-state lasers. IEEE J. Sel. Top. Quantum. Electron. 2, 435-453 (1996).

18. Kim, S. et al. High-mobility and low-power thin-film transistors based on multilayer $\mathrm{MoS}_{2}$ crystals. Nat. Commun. 3, 1011 (2012).

19. Mak, K. F., He, K., Shan, J. \& Heinz, T. F. Control of valley polarization in monolayer $\mathrm{MoS}_{2}$ by optical helicity. Nat. Nanotechnol. 7, 494-498 (2012).

20. Zeng, H., Dai, J., Yao, W., Xiao, D. \& Cui, X. Valley polarization in $\mathrm{MoS}_{2}$ monolayers by optical pumping. Nat. Nanotechnol. 7, 490-493 (2012).

21. Wang, S. et al. Broadband few-layer $\mathrm{MoS}_{2}$ saturable absorbers. Adv. Mater. 26, 3538-3544 (2014).

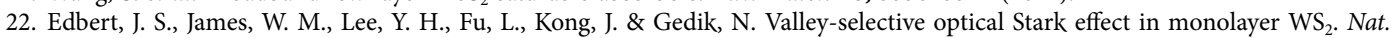
Mater. 14, 290-294 (2015).

23. Wu, W. et al. Piezoelectricity of single-atomic-layer $\mathrm{MoS}_{2}$ for energy conversion and piezotronics, Nat. 570, 470-474 (2014).

24. Yin, X. et al. Edge nonlinear optics on a $\mathrm{MoS}_{2}$ atomic monolayer. Science 344, 488-490 (2014).

25. Zhang, H. et al. Molybdenum disulfide $\left(\mathrm{MoS}_{2}\right)$ as a broadband saturable absorber for ultra-fast photonics. Opt. Express. 22, 7249-7260 (2014). 
26. Cho, W. B. et al. Boosting the nonlinear optical response of carbon nanotube saturable absorbers for broadband mode-locking of bulk lasers. Adv. Funct. Mater. 20, 1937-1943 (2010).

27. Bao, Q. L. et al. Atomic-layer graphene as a saturable absorber for ultrafast pulsed lasers. Adv. Funct. Mater. 19, 3077-3083 (2009).

28. Bonaccorso, F., Sun, Z., Hasan, T. \& Ferrari, A. C. Graphene photonics and optoelectronics. Nat. Photonics 4, 611-622 (2010).

29. Yu, H. et al. Topological insulator as an optical modulator for pulsed solid-state lasers. Laser Photonics Rev. 7, L77-L83 (2013).

30. Kumar, N. et al. Second harmonic microscopy of monolayer $M_{2}$. Phys. Rev. B 87, 161403(R) (2013).

31. Lee, C., Yan, H., Brus, L. E., Heinz, T. F., Hone, J. \& Ryu, S. Anomalous Lattice Vibrations of Single- and Few-Layer MoS ${ }_{2}$ ACS Nano 4, 2695-2700 (2010).

32. Kam, K. K. \& Parklnclon, B. A. Detailed photocurrent spectroscopy of the semiconducting group VIB transition metal dichalcogenides. J. Phys. Chem. 86, 463-467 (1982).

33. Enyashin, A., Gemming, S. \& Seifert, G. Nanosized allotropes of molybdenum disulfide. Eur. Phys. J.: Spec.Top. 149, 103-125 (2007).

34. Li, T. \& Galli, G. Electronic Properties of $\mathrm{MoS}_{2}$ Nanoparticles. J. Phys. Chem. C 111, 16192-16196 (2007).

35. Garmire, E. Resonant Optical Nonlinearities in Semiconductors. IEEE J. Sel. Top. Quantum Electron. 6, 1094-1110 (2000)

36. Zhang, X., Zhao, S., Wang, Q., Zhang, Q., Sun, L. \& Zhang, S. Optimization of $\mathrm{Cr}^{4+}$-doped saturable-absorber Q-switched lasers. IEEE J. Quantum Electron. 33, 2286-2294 (1997).

37. Nath, M., Govindaraj, A. \& Rao, C. N. R. Simple synthesis of $\mathrm{MoS}_{2}$ and $\mathrm{WS}_{2}$ nanotubes. Adv. Mater. 13, $283-286$ (2001).

\section{Acknowledgement}

The National Natural Science Foundation of China (Nos. 51025210, 51102156, 51422205 and 51272131), the Natural Science Foundation for Distinguished Young Scholars of Shandong Province (2014JQE27019) and Taishan Scholar Foundation of Shandong Province, China.

\section{Author Contributions}

H.Y. and H.Z. contributed equally to this work, conceived the experiments, performed the experiments, collected and analyzed the data, and wrote the paper; Y.Z. performed the experiments, collected and analyzed the data; S.W., Y.C. and L.M. prepared the saturable absorber; A.D. and M.T. provided the crystal samples; J.W. helped with the data analysis, theoretical calculation and paper writing.

\section{Additional Information}

Competing financial interests: The authors declare no competing financial interests.

How to cite this article: Zhang, Y. et al. Atomic-layer molybdenum sulfide optical modulator for visible coherent light. Sci. Rep. 5, 11342; doi: 10.1038/srep11342 (2015).

(c) (i) This work is licensed under a Creative Commons Attribution 4.0 International License. The images or other third party material in this article are included in the article's Creative Commons license, unless indicated otherwise in the credit line; if the material is not included under the Creative Commons license, users will need to obtain permission from the license holder to reproduce the material. To view a copy of this license, visit http://creativecommons.org/licenses/by/4.0/ 